\title{
Glucose levels as a prognostic marker in patients with ST-segment elevation myocardial infarction: a case-control study
}

\author{
Victoria Karetnikova ${ }^{1,2}$, Olga Gruzdeva', Evgenya Uchasova ${ }^{1 *}$, Anastasia Osokina ${ }^{1,2}$ and Olga Barbarash ${ }^{1,2}$
}

\begin{abstract}
Background: Patients with myocardial infarction (MI) have a high mortality. Therefore, new risk markers and predictors of an adverse outcome for $\mathrm{Ml}$ are required. The role of hyperglycemia in the development of cardiovascular complications in MI patients is still unclear.

Methods: A total of 529 consecutive patients with the diagnosis of ST-segment elevation acute coronary syndrome within $24 \mathrm{~h}$ of the onset of symptoms were included in the study. All of the patients underwent blood glucose measurement at admission to hospital. The glycemic profile, including measurement of blood glucose levels early in the night and in the morning ( 3 a.m. and 5 a.m.), was assessed in 77 patients with diabetes on days $6-10$ of the course of $\mathrm{Ml}$ to monitor the efficiency of blood glucose-lowering therapy and to detect hypoglycemic episodes.

Results: In-hospital mortality showed relationship between the level of blood glucose on admission and in-hospital mortality in patients with $\mathrm{MI}$ with ST-segment elevation in combination with diabetes mellitus. There was a direct linear relationship between blood glucose levels and in-hospital mortality in patients without diabetes.

Conclusion: Episodes of hypoglycemia recorded in Ml patients with diabetes in the hospital stage of treatment do not determine the prognosis, but enable identification of patients with an unfavorable course in the postinfarction period.
\end{abstract}

Keywords: Myocardial infarction, Diabetes mellitus, Blood glucose, Prognosis

\section{Background}

The role of hyperglycemia in the development of cardiovascular complications in myocardial infarction (MI) patients is still a matter of concern. There are two major perspectives on the role of hyperglycemia in the medical literature. One hypothesis is that hyperglycemia in patients with acute coronary syndrome (ACS) is induced by activation of adrenergic receptors. Another hypothesis is that hyperglycemia in patients with MI is a marker of pre-existing, but as yet undetected, carbohydrate metabolism disorders [1]. Recent studies have shown that hyperglycemia is often overlooked in $66 \%$ of patients admitted with acute MI, even though it meets the criteria for diabetes mellitus (DM) [2].

\footnotetext{
* Correspondence: evg.uchasova@yandex.ru

${ }^{1}$ Federal State Budgetary Institution "Research Institute for Complex Issues of

Cardiovascular Diseases", Kemerovo, the Russian Federation

Full list of author information is available at the end of the article
}

An association between high glucose levels in ACS patients and increased overall mortality has been shown [3]. Elevated blood glucose levels in ACS patients without DM are considered to be associated with a less favorable prognosis than in diabetic patients [4].

Hypoglycemia induced by adrenergic stimulation causes recurrent myocardial ischemia and triggers ventricular arrhythmias. Moreover, hypoglycemia inhibits metabolic processes in the myocardium and induces apoptosis in cardiomyocytes. The increased release of anti-insulin hormones results in the development of hyperglycemia, especially in patients suffering from short-course DM [5]. Therefore, alterations in blood glucose levels in patients with MI, regardless of diabetic status in the in-hospital period, may be regarded as a metabolic marker or a predictor of adverse outcomes. 
The present study aimed to assess the role of hyperand hypoglycemia in in-hospital and 1-year prognoses in patients with ST-segment elevation MI.

\section{Methods}

A total of 529 consecutive patients who were admitted to the Kemerovo Cardiology Dispensary in 2009 with a diagnosis of ST-segment elevation MI within $24 \mathrm{~h}$ of the onset of symptoms were included in the study. The exclusion criteria were as follows: MI complicating percutaneous coronary intervention or coronary artery bypass grafting surgery, end-stage renal disease (glomerular filtration rate $<30 \mathrm{~mL} / \mathrm{min}$ ), a positive history of diabetic coma, cancer pathology, and other diseases that greatly reduce life expectancy. 41 (7.9\%) patients revealed heart failure before, 124 (23.9 \%) had previous MI, 213 (41.0\%) smoked.

The study protocol was approved by the local ethics committee of the Federal State Budgetary Institution "Research Institute for Complex Issues of Cardiovascular Disease", developed in accordance with the WMA Declaration of Helsinki "Ethical principles for medical research involving human subjects" (amended in 2000) and "Rules for clinical practice in the Russian Federation", approved by the Ministry of Health of the Russian Federation on 19 June 2003. The study protocol was approved by the Local Ethics Committee of the Kemerovo Cardiology Dispensary. All patients provided written informed consent prior to their participation in the study.

ST-segment elevation MI was diagnosed according to the Russian Society of Cardiology (2007) as follows: chest pain lasting more than $20 \mathrm{~min}$; ST-segment elevation on electrocardiogram in two or more contiguous leads with the cut-off points of $\geq 0.1 \mathrm{mV}$ or complete left bundle branch block; elevated troponin $\mathrm{T}$ levels $\geq 0.1 \mathrm{ng} / \mathrm{mL}$ and/or creatinine kinase-MB levels $>25 \mathrm{IU} / \mathrm{L}$. The presence of at least two criteria, including elevated biochemical markers of myocardial necrosis, was considered sufficient for diagnosis of STsegment elevation MI.

Repeated measurement of blood glucose levels and an oral glucose tolerance test (OGTT), performed on days 10-14 in the in-hospital period, were used to diagnose type $2 \mathrm{DM}$ in patients who had unrecognized diabetes. A positive history of DM was confirmed by examination of medical records.

Diabetes was diagnosed when two or more independent determinations of glucose levels on different days were $\geq 7.0 \mathrm{mmol} / \mathrm{l}(126 \mathrm{mg} / \mathrm{dL})$. In doubtful cases of diabetes, such as the characteristic clinical picture of diabetes and a fasting plasma glucose within $6.6-7.7 \mathrm{mmol} / \mathrm{l}$, an OGTT was performed. An OGTT $\geq 11.1 \mathrm{mmol} / \mathrm{l}$ was diagnosed as type $2 \mathrm{DM}$. In addition, type $2 \mathrm{DM}$ was considered when HbA1c was $\geq 6.5 \%$ (according to the recommendations of the American Diabetes Association).

All of the patients underwent blood glucose measurement at admission to hospital. Moreover, the glycemic profile, including measurement of blood glucose levels in the early night and morning ( 3 a.m. and 5 a.m.) was assessed in 77 patients with diabetes on days 6-10 of the course of MI to monitor the efficiency of blood-glucoselowering therapy and to detect hypoglycemic episodes. During the in-hospital period, patients were treated according to the Russian Society of Cardiology guidelines for diagnosis and treatment of patients with acute STsegment elevation MI (2007).

The endpoints of prognosis at 1 year were overall and cardiovascular mortality, recurrent $\mathrm{MI}$, progression of angina pectoris, decompensated heart failure, acute stroke, hospitalization for acute coronary events, and urgent repeated revascularization. Achievement of these endpoints was evaluated as an unfavorable outcome. The survival rate and time to onset of adverse events were assessed.

\section{Assays}

Blood serum and plasma were collected. Serum was separated from venous blood by centrifugation at $3000 \times g$ for $20 \mathrm{~min}$ and stored at $-70{ }^{\circ} \mathrm{C}$. Glucose levels were measured using a standard Thermo Fisher Scientific test system (Thermo Fisher Scientific Oy, Vantaa, Finland) with a Konelab 30i biochemistry analyzer (Thermo Fisher Scientific Oy).

\section{Statistical analysis}

The data were processed using the statistical software package Statistica 6.0 (InstallShield Software Corp., Chicago, IL, USA). Results are presented as the median (Me) and $25 \%$ and $75 \%$ quartiles of the Me (Q1;Q3). Time to onset of adverse events was measured using the Cox regression model. $p<0.05$ was considered statistically significant.

\section{Results}

Blood glucose levels at admission were measured in 520 out of 529 patients. A total of 99 (19.03\%) patients had type 2 DM, of whom 11 (11.11 \%) had newly diagnosed DM within the in-hospital period. DM was diagnosed in four $(36.36 \%)$ patients according to the results of the OGTT and in seven (63.64\%) patients based on glucose levels determined within a day. Patients with diabetes were significantly older than non-diabetic patients $(p=0.002)$. Female patients with DM (63.0\%) were more prevalent than female patients without DM $(28.67 \%, p=0.001)$. There was a higher rate of obesity (75.0\%), as well as hypertension (96.0\%), angina pectoris $(64.0 \%)$, previous MI (38.0\%), congestive heart 
failure (19.0\%), and hypercholesterolemia (20.0\%), in patients with DM than in those without DM $(p<0.05)$. Patients with diabetes had a significantly higher average Thrombolysis In Myocardial Infarction risk score (5.0) than those without DM (3.0, $p=0.001)$. The in-hospital mortality rate was higher in patients with diabetes $(21.05 \%)$ than in non-diabetic patients $(12.57 \%, p=0.008)$.

To evaluate the effects of blood glucose levels on inhospital mortality, all patients, regardless of diabetic status, were assigned to three groups according to blood glucose levels at admission. One group included MI patients with euglycemia on admission (blood glucose levels $<7.0 \mathrm{mmol} / \mathrm{L}$ ), a second group included patients with moderate hyperglycemia (7.1-9.0 $\mathrm{mmol} / \mathrm{L})$, and a third group included patients with severe hyperglycemia ( $>9.0 \mathrm{mmol} / \mathrm{L})$. Blood glucose levels at admission as a criterion for group assignment was adopted from the clinical trial the Japanese Acute Coronary Syndrome Study, which aimed to assess the prognostic value of hyperglycemia [6].

The clinical and demographic data of MI patients with and without DM are shown in Tables 1 and 2 .

The highest rate of in-hospital mortality (20.0\%) was found in patients with glycemia on admission, corresponding to euglycemia, whereas the lowest rate of mortality rate $(11.1 \%)$ was found in patients with moderate hyperglycemia.

Non-diabetic MI patients with euglycemia on admission demonstrated the lowest rate of in-hospital mortality $(2.7 \%)$, whereas the highest rate was observed in the group of patients with severe hyperglycemia $(20.6 \%, p<0.001)$. Therefore, an increase in blood glucose levels by $1 \mathrm{mmol} / \mathrm{L}$ in MI patients without diabetes at admission was associated with a $10.1 \%$-fold increase in in-hospital mortality.

Comparative analysis of blood glucose levels at admission between patients with a different 1-year prognosis showed that non-diabetic patients with a poor prognosis had significantly higher blood glucose levels than nondiabetic patients. Patients with diabetes with a poor prognosis also had higher blood glucose levels than nondiabetic patients, but this was not significant.

Fasting glucose levels, measured on days 10-14 from the onset of MI, were similar in patients with DM and those without DM with a poor prognosis and high blood glucose levels $(p>0.05)$. Assessment of the glycemic profile, including measurement of blood glucose levels in the early night and morning hours (3 a.m. and 5 a.m.) showed that $27(35.07 \%)$ patients with diabetes suffered from hypoglycemic episodes (blood glucose levels $<3.5 \mathrm{mmol} / \mathrm{L}$ ).

Initiated hypoglycemic therapy, including administration of insulin, was not significantly different between the groups of patients with and without episodes of hypoglycemia. Insulin was administered in five (18.52\%) patients with hypoglycemic episodes and in seven (14.00\%) patients without hypoglycemia.

Notably, in MI patients, hypoglycemic status did not affect the number of in-hospital complications. However, patients with hypoglycemia reported more frequent $(p>0.05)$ development of left ventricular aneurysm and early post-infarction angina. There were no significant differences in the mortality rate and the incidence rate of ventricular arrhythmias in patients with and without hypoglycemia.

With regard to significant hypoglycemic episodes, as a marker of 1-year unfavorable prognosis in MI patients, patients with hypoglycemia were more likely to have decompensated heart failure and new coronary acute events (recurrent $\mathrm{MI}$ and progressive angina) during the 1-year follow-up period (Table 3). Therefore, hypoglycemia appeared to have a higher predictive value than a clinical value. Overall, there were no cases with a favorable prognosis among patients with

Table 1 Clinical and demographic characteristics of diabetic patients with myocardial infarction according to blood glucose levels

\begin{tabular}{|c|c|c|c|c|c|}
\hline \multirow[t]{2}{*}{ Variable } & \multirow{2}{*}{$\begin{array}{l}\text { All diabetic } \\
\text { patients }\end{array}$} & \multicolumn{4}{|l|}{ Diabetic patients } \\
\hline & & Euglycemia & Moderate hyperglycemia & Severe hyperglycemia & $p$ \\
\hline Number of patients, n (\%) & 99 & $5(5.05)$ & $9(9.09)$ & $85(85.85)$ & \\
\hline Age, years & $68.0(59.0-74.0)$ & $68.0(63.0-76.0)$ & $63.0(53.0-71.0)$ & $68.0(59.0-73.0)$ & $>0.05$ \\
\hline Males, n (\%) & $37(37.37)$ & $1(20.0)$ & $3(33.33)$ & $33(38.82)$ & $>0.05$ \\
\hline $\mathrm{AH}, \mathrm{n}(\%)$ & $95(95.95)$ & $5(100.0)$ & $8(88.88)$ & $82(96.47)$ & $>0.05$ \\
\hline Killip II - IV, n (\%) & $40(40.40)$ & $3(60.0)$ & $3(33.33)$ & $34(40.0)$ & $>0.05$ \\
\hline Time before admission, hour & $4.72(2.4 ; 7.7)$ & $4.0(1.8 ; 10.1)$ & $9.3(5.4 ; 15.6)$ & $3.6(1.4 ; 6.7)$ & $>0.05$ \\
\hline Reperfusion, n (\%) & $52(52.52)$ & $4(80.0)$ & $4(44.44)$ & $44(51.76)$ & $>0.05$ \\
\hline Thrombolysis, n (\%) & 19 (19.19) & $1(20.0)$ & $1(11.11)$ & $17(20.00)$ & $>0.05$ \\
\hline $\mathrm{PCl}, \mathrm{n}(\%)$ & $42(42.42)$ & $3(60.0)$ & $3(33.33)$ & $36(42.35)$ & $>0.05$ \\
\hline Admission blood glucose, mol/L & $15.0(11.30-20.0)$ & $6.20(6.20-6.40)$ & $8.30(7.43-8.50)$ & $13.80(7.04-21.03)$ & $P_{2-3}<0.0001^{*}$ \\
\hline
\end{tabular}

*Mann-Whitney test 
Table 2 Clinical and demographic characteristics of non-diabetic patients with myocardial infarction according to blood glucose levels

\begin{tabular}{|c|c|c|c|c|c|}
\hline \multirow[t]{2}{*}{ Variable } & \multirow{2}{*}{$\begin{array}{l}\text { All non- } \\
\text { diabetic } \\
\text { patients }\end{array}$} & \multicolumn{4}{|c|}{ Non-diabetic patients } \\
\hline & & Euglycemia & Moderate hyperglycemia & Severe hyperglycemia & $p$ \\
\hline Number of patients, n (\%) & 421 & $183(43.46)$ & $141(33.49)$ & $97(23.0)$ & \\
\hline \multirow[t]{2}{*}{ Age, years } & $61.0(53.0-72.0)$ & $58.0(51.0-69.0)$ & $61.0(55.0-73.0)$ & 69.0(61.0-76.0) & $P_{1-2}<0.0001^{*}$ \\
\hline & & & & & $P_{1-3}<0.0001^{*}$ \\
\hline \multirow[t]{2}{*}{ Males, n (\%) } & $300(71.25)$ & $143(78.14)$ & $98(69.50)$ & $59(60.82)$ & $P_{1-2}=0.05^{* *}$ \\
\hline & & & & & $P_{1-3}=0.001^{* *}$ \\
\hline $\mathrm{AH}, \mathrm{n}(\%)$ & $367(87.17)$ & 165 (90.16) & $116(82.26)$ & $86(88.65)$ & $P_{1-2}=0.02^{* *}$ \\
\hline \multirow[t]{3}{*}{ Killip II - IV, n (\%) } & $94(22.32)$ & $22(12.02)$ & $28(19.85)$ & $44(45.36)$ & $P_{1-2}=0.03^{* *}$ \\
\hline & & & & & $P_{1-3}<0.0001^{* *}$ \\
\hline & & & & & $P_{2-3}<0.0001^{* *}$ \\
\hline Time before admission, hour & $3.1(1.9 ; 6.6)$ & $3.0(1.8 ; 8.0)$ & $3.1(2.0 ; 6.2)$ & $3.4(2.0 ; 5.5)$ & $>0.05$ \\
\hline Reperfusion, n (\%) & $250(59.38)$ & $102(55.73)$ & $88(62.41)$ & $60(61.85)$ & $>0.05$ \\
\hline \multirow[t]{2}{*}{ Thrombolysis, n (\%) } & $72(17.10)$ & $18(9.83)$ & $34(24.11)$ & $20(20.61)$ & $P_{1-2}=0.0005^{* *}$ \\
\hline & & & & & $P_{1-3}=0.01^{* *}$ \\
\hline $\mathrm{PCl}, \mathrm{n}(\%)$ & $213(50.59)$ & $93(50.81)$ & $69(48.93)$ & $51(52.57)$ & $>0.05$ \\
\hline \multirow[t]{2}{*}{ Admission blood glucose, $\mathrm{mol} / \mathrm{L}$} & $7.20(6.04-8.72)$ & $5.90(5.21-6.4)$ & $7.80(7.37-8.22)$ & $10.60(6.33-12.70)$ & $P_{1-3}=0.003^{* *}$ \\
\hline & & & & & $P_{2-3}=0.001^{*}$ \\
\hline
\end{tabular}

*Mann-Whitney test; **Fisher's test

hypoglycemia during the 1-year follow-up period. Importantly, patients with hypoglycemia had a short time to onset of adverse events (Table 4).

\section{Discussion}

The current findings are consistent with the results of previous registries, suggesting $14.3-40.9 \%$ prevalence of DM in patients with acute myocardial infarction according to the national population characteristics [6, 7]. However, all registries show a significant increase in the in-hospital mortality rate in diabetic patients with myocardial infarction, compared with patients without carbohydrate metabolism disorders. The GRACE registry reported that patients with ST-segment elevation myocardial infarction and previously diagnosed diabetes demonstrated $11.7 \%$ in-hospital mortality, whereas patients without carbohydrate metabolism disorders had $6.4 \%$ mortality rate [6]. The results of the GulfRACE register also indicated a significant increase in the in-hospital mortality rate among diabetic patients with acute coronary syndrome compared with patients without diabetes ( $4.4 \%$ vs. $3.4 \%$, respectively, $p<0.01)[7,8]$.
The present study showed different relationships between blood glucose levels at admission and in-hospital mortality in MI patients with and without diabetes. Therefore, blood glucose levels $<7.0 \mathrm{mmol} / \mathrm{L}$ at admission in MI patients without diabetes were associated with the lowest in-hospital mortality rate, and linearly increased with increased blood glucose levels. The lowest rate of in-hospital mortality was found in patients with moderate hyperglycemia. A higher rate of inhospital mortality in this group of patients was associated with severe hyperglycemia and euglycemia. Notably, severe hyperglycemia on admission was associated with increased in-hospital mortality in MI patients with and without DM.

When analyzing the association between diabetic patients with MI and euglycemia on admission to hospital and high in-hospital mortality, there is a negative effect of hypoglycemia on the prognosis in these patients [9]. However, patients with diabetes tend to adapt better to higher blood glucose levels than to normal levels. Therefore, euglycemia (diagnosed on admission) may be

Table 3 Prognostic significance of hypoglycemic episodes

\begin{tabular}{llll}
\hline Endpoints (after 1 year) & Patients with hypoglycemia, $n(\%) ; n=27$ & Patients without hypoglycemia, $n(\%) ; n=50$ & $p(10.00 \%)$ \\
\hline Death & $4(14.82 \%)$ & $10(20.00 \%)$ & $\geq 0.05$ \\
Recurrent Ml & $8(29.63 \%)$ & $9(18.00 \%)$ & 0.034 \\
Decompensated CHF & $9(33.33 \%)$ & $6(12.00 \%)$ & 0.000 \\
Unstable angina & $11(40.74 \%)$ & 0.000 \\
\hline
\end{tabular}


Table 4 Time to onset of adverse events in diabetic patients with $\mathrm{Ml}$ and hypoglycemic episodes

\begin{tabular}{llll}
\hline Groups of patients & Patients without adverse events, $n$ & Time-to-onset, $\mathrm{Me}$, the 25th and 75th percentiles & $95 \% \mathrm{Cl}$ \\
\hline with hypoglycemia & 0 & $2(1-6)$ month & $1.98-4.73$ \\
without hypoglycemia & $6(10.2 \%)$ & $3(1-6)$ month & $2.71-5.05$
\end{tabular}

regarded as relative hypoglycemia affecting the prognosis. Consequently, chronic hyperglycemia (estimated on admission and on days 10-14 of the in-hospital period) could be an important marker of poor prognosis in MI patients with and without DM.

Comparative assessment of the effect of glycemic status on the prognosis in patients with MI should consider postprandial glucose levels, which play an important role in the development of adverse cardiovascular events $[10,11]$. The DECODE study [12], which assessed the risk of death under different variants of hyperglycemia, showed that the postprandial glucose level is an independent risk factor, with higher prognostic significance than glycated hemoglobin levels. Several studies have suggested that after-meal high blood glucose levels are more important for the risk assessment of cardiovascular complications in diabetic patients than fasting glucose levels [12]. Therefore, assessment of the effects of acute and chronic hyperglycemia on induction of oxidative stress in patients with DM suggests that its main triggering mechanism is acute deviations in blood glucose levels, but not long-term chronic hyperglycemia (or small fluctuations in postprandial blood glucose) [13]. Postprandial hyperglycemia in patients with DM is associated with reduced myocardial perfusion caused by microvascular abnormalities. We have previously shown a positive effect of adequate glycemic control [14].

In general, hyperglycemia on admission in ACS patients is a major predictor of survival and increased risk of development of cardiovascular events, regardless of diabetic status [1]. Moreover, the present study showed that hyperglycemia on admission to hospital, as an indicator reflecting the degree of oxidative stress in acute coronary events, significantly affects immediate and long-term prognosis in patients with MI. However, the majority $(74.2 \%)$ of patients with DM had high levels of postprandial and fasting glucose (i.e., presence of chronic hyperglycemia), which affected the prognosis during the in-hospital period. Notably, the prognostic value of hyperglycemia did not change during the whole in-hospital period [15]. Nevertheless, previous studies have shown an increase in the mortality rate in patients with hypoglycemia and MI [15-17].

Taking into consideration the adverse effect of hypoglycemic episodes on the long-term prognosis in MI patients with DM, spontaneous hypoglycemia might be a marker of the severity of disease and adverse outcomes or an independent predictor of a poor prognosis. Further studies are required to determine the role of hypoglycemia as a mediator of adverse outcomes in MI patients.

\section{Conclusions}

Hyperglycemia, assessed in patients with MI with and without DM when they are admitted to hospital, affects the short and long-term prognosis. Hyperglycemia and the in-hospital prognosis show a linear relationship in patients without DM. The presence of hypoglycemia in patients with DM does not affect the in-hospital prognosis, but enables determination of patients with an unfavorable course in the postinfarction period.

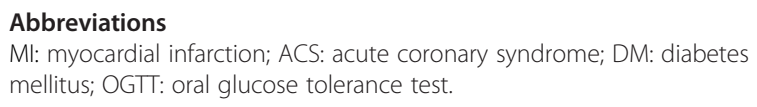

\section{Acknowledgements}

The authors wish to thank Catherine Anikeeva for assistance in writing this article.

\section{Availability of data and materials}

Not applicable.

\begin{abstract}
Authors' contributions
VK and OG were principal investigators and study coordinators. They participated in all stages of recruitment of the patients and in analysis of the data, and drafted and critically reviewed the manuscript. EU and AO participated in all stages of recruitment of the patients and critically reviewed the manuscript. OB was a principal investigator. All other study investigators conducted the study and collected the data. All authors read and approved the final manuscript.
\end{abstract}

\section{Ethical approval}

The study protocol was approved by the local ethics committee of the Federal State Budgetary Scientific Institution Research Institute for Complex Issues of Cardiovascular Diseases.

\section{Consent for publication}

Not applicable.

\section{Competing interests}

This manuscript has been read and approved by all the authors. This paper is unique and is not under consideration by any other journal and has not been published elsewhere. The authors of this paper report no conflicts of interest. The authors confirm that they have permission to reproduce any copyrighted material.

\section{Author details}

${ }^{1}$ Federal State Budgetary Institution "Research Institute for Complex Issues of Cardiovascular Diseases", Kemerovo, the Russian Federation. 'Federal State Budget Educational Institution of Higher Professional Education "Kemerovo State Medical Academy" the Ministry of Health of the Russian Federation, Kemerovo, the Russian Federation.

Received: 12 July 2015 Accepted: 16 May 2016

Published online: 01 June 2016 


\section{References}

1. Kobalava JD, Tolkachev W. Hyperglycemia in patients with acute coronary syndrome: state of the art. Scientific Committee's recommendations on diabetes by the American Heart Association. Kardiologiia. 2009;3:77-85.

2. Conaway DG, O'Keefe $\mathrm{JH}$, Reid KJ. Frequency of undiagnosed diabetes mellitus in patients with acute coronary syndrome. Am J Cardiol. 2005;96:363-5.

3. Meier JJ, Deifuss S, Klamann A, Launhardt V, Schmiegel WH, Nauck MA. Plasma glucose at hospital admission and previous metabolic control determine myocardial infarct size and survival in patients with and without type 2 diabetes: the Langendreer Myocardial Infarction and Blood Glucose in Diabetic Patients Assessment (LAMBDA). Diabetes Care. 2005;28(10):2551-3.

4. Stranders I, Diamant M, van Gelder RE, Spruijt HJ, Twisk JW, Heine RJ, Visser FC. Admission blood glucose level as risk indicator of death after myocardial infarction in patients with and without diabetes mellitus. Arch Intern Med. 2004;164(9):982-8.

5. Abbate A, Biondi-Zoccai GL. The difficult task of glycaemic control in diabetics with acute coronary syndromes: finding the way to normoglycaemia avoiding both hyper- and hypoglycaemia. Eur Heart J. 2005;26(13):1245-8.

6. Ishihara M, Kojima S, Sakamoto T, Asada Y, Tei C, Kimura K, et al. On behalf of the Japanese Acute Coronary Syndrome Study (JACSS) Investigators. Acute hyperglycemia is associated with adverse outcome after acute myocardial infarction in the coronary intervention era. Am Heart J. 2005;150:814-20.

7. Rydén L, Standl E, Bartnik M, Van den Berghe G, Betteridge J, de Boer MJ, Cosentino F, et al. Guidelines on diabetes, pre-diabetes and cardiovascular diseases: executive summary. Eur Heart J. 2007;28:88-136

8. Zubaid M. Preliminary results from Gulf Registry of Acute Coronary Events (GulfRACE). Heart Views. 2007-2008; 8(4): 155-8.

9. Desouza C, Salazar H, Cheong B, Murgo J, Fonseca V. Association of hypoglycaemia and cardiac ischaemia: a study based on continuous monitoring. Diabetes Care. 2003;26:1485-9.

10. Ceriello A. Postprandial hyperglycaemia: a new risk factor for cardiovascular disease. Diabet Metab Heart. 2008;17:363-73.

11. Raguso CA, Helary C, Philippe J. Importance of the postprandial glycemia in the management of type 2 diabetes. Rev Med Suisse. 2008:4(160):1383-6.

12. Is fasting glucose sufficient to define diabetes? Epidemiological data from 20 European studies. The DECODE-study group. European Diabetes Epidemiology Group. Diabetes Epidemiology: Collaborative analysis of Diagnostic Criteria in Europe. Diabetologia. 1999;42(6):654-74.

13. Monnier L, Mas E, Ginet C, Michel F, Villon L, Cristol JP, Colette C. Activation of oxidative stress by acute glucose fluctuation compared with sustained chronic hyperglycemia in patients with type 2 diabetes. JAMA. 2006;295:1681-7.

14. Scognamiglio R, Negut C, de Kreutzenberg SV, Tiengo A, Avogaro A. Effects of different insulin regimes on postprandial myocardial perfusion defects in type 2 diabetic patients. Diabetes Care. 2006;29(1):95-100.

15. Kosiborod M, Inzucchi SE, Krumholz HM, Xiao L, Jones PG, Fiske S, et al. Glucometrics in patients hospitalized with acute myocardial infarction: defining the optimal outcomes-based measure of risk. Circulation. 2008; 117(8):1018-27.

16. Svensson AM, McGuire DK, Abrahamsson P, Dellborg M. Association between hyper- and hypoglycaemia and 2 year all-cause mortality. Eur Heart J. 2005;26(13):1255-61.

17. Pinto DS, Skolnick AH, Kirtane AJ, Murphy SA, Barron HV, Giugliano RP, TIMI Study Group, et al. U-shaped relationship of blood glucose with adverse outcomes among patients with ST-segment elevation myocardial infarction. J Am Coll Cardiol. 2005;46(1):178-80.

\section{Submit your next manuscript to BioMed Central and we will help you at every step:}

- We accept pre-submission inquiries

- Our selector tool helps you to find the most relevant journal

- We provide round the clock customer support

- Convenient online submission

- Thorough peer review

- Inclusion in PubMed and all major indexing services

- Maximum visibility for your research

Submit your manuscript at www.biomedcentral.com/submit
Biomed Central 